\title{
Review Article \\ Detection of Paroxysmal Atrial Fibrillation in Stroke/Tia Patients
}

\author{
Muhib Khan ${ }^{1}$ and Daniel J. Miller ${ }^{2}$ \\ ${ }^{1}$ Department of Neurology, University of Massachusetts, USA \\ ${ }^{2}$ Department of Neurology, Henry Ford Hospital, USA \\ Correspondence should be addressed to Muhib Khan; muhibalamkhan@hotmail.com
}

Received 25 January 2013; Accepted 4 March 2013

Academic Editor: V. Padma

Copyright (C) 2013 M. Khan and D. J. Miller. This is an open access article distributed under the Creative Commons Attribution License, which permits unrestricted use, distribution, and reproduction in any medium, provided the original work is properly cited.

\begin{abstract}
One-third of stroke and transient ischemic attack (TIA) are cryptogenic, and paroxysmal atrial fibrillation (PAF) has been suggested as a possible cause for these cryptogenic strokes. Multiple studies have recently evaluated long-term cardiac rhythm monitoring with good yield for PAF. The duration of monitoring varies between studies as well as the qualifying event definition. Moreover, the clinical significance of very brief atrial fibrillation events is unclear in the literature. This paper provides an overview of current advances in the detection of paroxysmal atrial fibrillation, the clinical and genetic factors predictive of arrhythmia detection, and the therapeutic dilemma concerning this approach.
\end{abstract}

\section{Introduction}

One-third of stroke and transient ischemic attack (TIA) are cryptogenic requiring additional investigation and intervention [1]. Occult paroxysmal atrial fibrillation (PAF) has been suggested as a possible cause for these cryptogenic strokes [2]. Atrial fibrillation has been long associated with high risk of stroke, but most of this knowledge is derived from patient data from chronic atrial fibrillation. It has been suggested that PAF is more prevalent than persistent atrial fibrillation in stroke and TIA patients [3]. Anticoagulation therapy initiated after detection of atrial fibrillation (AF) provides an additional $40 \%$ risk reduction of stroke as compared to antiplatelet therapy alone [4].

Therefore, it is important to diagnose AF after an ischemic stroke to provide maximal stroke prevention therapy. Current standard of care dictates an admission electrocardiogram (ECG) and at least $24 \mathrm{~h}$ of continuous telemetry monitoring [5]. However, brief asymptomatic paroxysmal atrial fibrillation events may remain undetected by traditional methods of screening. Recent technological advances have made it possible to perform long-term cardiac rhythm monitoring up to months or even years after a stroke.

\section{Definition}

Paroxysmal atrial fibrillation is not clearly defined in the literature. There is controversy over the duration and morphology of the ECG data in defining an event qualifying for atrial fibrillation. Studies evaluating the incidence of PAF in stroke and TIA patient populations have used different definitions adding confusion about the true incidence. In our paper, we have highlighted the need for a rigorous definition of paroxysmal atrial fibrillation especially in the light of widely used advanced rhythm monitoring devices.

\section{Epidemiology}

Atrial fibrillation prevalence is associated with age with $0.5 \%$ at $50-59$ years of age increasing to $8.8 \%$ at $80-89$ years [2]. PAF comprises from $25 \%$ to $60 \%$ of these cases [2]. The true incidence of PAF is unclear as most of the prevalence studies used symptomatic events, and prolonged rhythm monitoring was not available at the time on these studies. Moreover, again the variable definitions might have an impact of the incidence reported. 
TABLE 1: Yield of long-term cardiac rhythm monitoring studies.

\begin{tabular}{|c|c|c|c|c|c|}
\hline Study & Patient population & Duration (days) & Sample size & No. diagnosed & Percentage \\
\hline Barthélémy et al. 2003 [6] & Stroke/TIA & 4 & 28 & 4 & 14.3 \\
\hline Sposato et al. 2012 [7] & Stroke/TIA & 5 & 155 & 21 & 13.5 \\
\hline Stahrenberg et al. 2010 [8] & Stroke/TIA & 7 & 220 & 28 & 12.7 \\
\hline Jabaudon et al. 2004 [9] & Stroke/TIA & 7 & 88 & 5 & 5.7 \\
\hline Tayal et al. 2008 [10] & Stroke/TIA & 21 & 56 & 13 & 23 \\
\hline Miller et al. 2013 [11] & Stroke/TIA & 21 & 156 & 27 & 17.3 \\
\hline Bhatt et al. 2011 [12] & Stroke/TIA & 21 & 62 & 15 & 24 \\
\hline Elijovich et al. 2009 [13] & Stroke/TIA & 30 & 20 & 4 & 20 \\
\hline Gaillard et al. 2010 [14] & Stroke/TIA & 30 & 98 & 9 & 9.2 \\
\hline Flint et al. 2012 [15] & Stroke & 30 & 239 & 29 & 12.1 \\
\hline Ziegler et al. 2010 [16] & Stroke/TIA & 365 & 163 & 45 & 28 \\
\hline Total & & & 1285 & 200 & 15.5 \\
\hline
\end{tabular}

\section{Pathophysiology}

Atrial arrhythmias have varied pathophysiology ranging from rapidly discharging foci, microreentry, macroreentry, and autonomic modulation of the atria. These processes are usually due to structural abnormality within the atria. This leads to mechanical dysfunction with resultant thrombus formation in the complex, pectinate-rich structure of the left atrial appendage.

\section{Natural History}

Paroxysmal atrial fibrillation is a self-promoting process, and if these events are left untreated, they can progress to persistent atrial fibrillation [3]. The goal of therapy is to maintain sinus rhythm and appropriate anticoagulation [3].

\section{Studies}

Multiple studies have been published recently highlighting higher yield of atrial fibrillation detection with longer monitoring and newer devices [6-16]. The yield varied (5\%$28 \%$ ) depending on the choice of monitoring devices, study population, stroke characteristics, interval of monitoring, from stroke onset, duration of cardiac monitoring and most importantly the definition of paroxysmal atrial fibrillation. Table 1 provides a snap shot of these studies.

Most of these studies defined paroxysmal atrial fibrillation as events lasting more than 30 seconds. The 30 seconds benchmark used to describe atrial fibrillation events comes from the AHA 2006 guidelines [17]. It is unclear from the manuscript how the authors came up with the 30 seconds benchmark although they do mention that shorter events may be relevant in the right clinical setting [17]. It has been suggested that the duration of atrial fibrillation events with higher specificity can be up to 5 minutes. But then again, this higher specificity comes at the cost of lower sensitivity, and a critical balance between the two needs to be established [18].

It is also to be kept in mind that most of these studies are hospital-based identification of stroke/TIA patients with prolonged rhythm monitoring at the discretion of treating physician, and the data was analyzed retrospectively. Therefore, these studies are not free from selection bias.

It has been shown that these event monitors do have a high false positive rate especially for short events. More refined software with better algorithms to filter out myopotential artifacts can increase specificity, but there is always the risk of losing the sensitivity of event detection [19].

This brings us to the question of what duration of atrial fibrillation events is predictive of a future stroke. No clear association between the duration of events and stroke risk has been established in the literature. It has been shown that even excessive supraventricular ectopic beats not constituting atrial fibrillation increase ischemic stroke and atrial fibrillation detection rates [20]. It is unclear how often these short atrial fibrillation events lead to chronic atrial fibrillation. However, it has also been noted that higher ( $>5.5$ hours) burden of atrial fibrillation events (total duration) leads to a higher stroke risk although this total duration was extrapolated from a long duration of monitoring (>365 days) [21]. Additionally, a recent study showed increased ischemic stroke rates, as well as verified atrial fibrillation rates, in patients that were found to have subclinical atrial tachyarrhythmias of $>6$ mins [22].

The ongoing study CRYSTAL AF is investigating the value of longer-term monitoring with an implantable loop recorder in patients with cryptogenic stroke to identify the predictive value of these events. Moreover, both CRYSTAL AF and IMPACT trials will help us to identify the best candidates for anticoagulation $[23,24]$. IMPACT is also evaluating the therapeutic intervention implied after detection of these events which is the final goal of any diagnostic evaluation in secondary stroke prevention.

\section{Predictors}

Multiple predictors of PAF in cryptogenic stroke patients have been proposed by these studies including diabetes, female gender, premature atrial complexes (PACs) on ECG, left atrial dilatation, left ventricular reduced ejection fraction (EF), higher stroke severity assessed by National Institute of Health Stroke Scale (NIHSS), nonlacunar anterior circulation 
TABle 2: Predictors of atrial fibrillation detection in patients with cryptogenic stroke.

\begin{tabular}{ll}
\hline Study & $\begin{array}{l}\text { Predictors of atrial fibrillation } \\
\text { detection }\end{array}$ \\
\hline Tayal et al. 2008 [10] & Diabetes \\
\hline Miller at al. 2013 [11] & $\begin{array}{l}\text { Female gender } \\
\text { PAC on ECG } \\
\text { Left atrial dilatation } \\
\text { Left ventricular EF reduction } \\
\text { NIHSS }\end{array}$ \\
\hline Gaillard et al. 2010 [14] & N100 PACs on 24 hr Holter \\
& $\begin{array}{l}\text { Nonlacunar anterior circulation } \\
\text { acute infarcts }\end{array}$ \\
\hline \multirow{2}{*}{ Bhatt et al. 2011 [12] } & Multiple acute infarcts \\
& Premature ventricular complexes \\
\hline
\end{tabular}

infarcts on neuroimaging, cortical infarcts on neuroimaging, and congestive heart failure [10-12, 14]. Of note, symptoms are not a significant predictor of detecting PAF as most of these events are asymptomatic [25]. Table 2 provides a synopsis of these predictors.

Most of these predictors make pathophysiological sense because of their ability to modify the atrial rhythms eventually leading to atrial fibrillation. Dilated left atrium and premature atrial complexes stand out as the most important predictors. Transthoracic echocardiogram parameters are useful in selecting the patients for prolonged monitoring in this regard [26]. Premature atrial complexes (PACs) are an important predictor, and a careful analysis of admission ECG should be performed on all stroke patients to identify these PACs for long-term monitoring selection [27].

These various predictors have been consolidated into risk assessment scoring schemes to provide a predictive model for PAF detection [28, 29]. These scores are useful tools, but clinical judgment should always be implied in selecting patients for long-term monitoring.

\section{Future Direction}

There is a novel concept of atrial fibrillation density which implies temporal clustering of atrial fibrillation events in a short period of time. Essentially, a patient who is monitored in the time window when most of these events are clustering has a higher probability of being detected with monitoring devices as compared to a window period when the events are not clustered. This concept again reaffirms the need to monitor these patients for longer duration [30].

The new subcutaneous implantable cardiac monitors (REVEAL) have shown potential to detect paroxysmal atrial fibrillation with higher patient compliance [31] and longer duration of monitoring. One attractive feature of this device is its MRI compatibility [32].

So far, there has been only one randomized clinical trial performed to evaluate the impact of prolonged monitoring on therapeutic decisions. The trial failed to show any benefit of long-term monitoring over routine clinical followup but the sample size of this trial was very small, and the findings need to be validated in a larger clinical study [33].

The iPhone $4 \mathrm{~S}$ has been shown to potentially act as a monitoring device for detecting PAF and has broad worldwide implications as a method of bringing monitoring to the masses [34].

Multiple genetic polymorphisms have been implicated in pathogenesis of atrial fibrillation such as PITX2, ZFHX3, KCNN3, PRRX1, CAV1, SYNE2, FBP, HCN4, SYNPO2L, and MYOZ1.

These genes mostly encode for proteins important in the integrity of cardiac myocyte structure and normal physiology [35]. It has been noted that these genetic markers do not increase the detection yield of atrial fibrillation when added to conventional risk factors for atrial fibrillation. Further studies are needed to establish the clinical implication of genetic testing for atrial fibrillation [36].

\section{Conclusions}

Long-term cardiac rhythm monitoring plays an increasingly important role in determining the etiology of stroke/TIA. The widespread use of these monitors will increase the incidence of PAF, raising questions about management of these events. Future studies should focus on the optimal duration of monitoring, predictors of detecting atrial fibrillation, stroke risk pertinent to paroxysmal atrial fibrillation, and the duration of these events. There is also a need to study how often the short atrial fibrillation events lead to chronic atrial fibrillation and optimal treatment strategy, either antiplatelet or anticoagulation, in patients exhibiting brief events.

\section{Conflict of Interests}

The authors do not have any conflict of interests with the content of the paper.

\section{References}

[1] P. L. Kolominsky-Rabas, M. Weber, O. Gefeller, B. Neundoerfer, and P. U. Heuschmann, "Epidemiology of ischemic stroke subtypes according to TOAST criteria: incidence, recurrence, and long-term survival in ischemic stroke subtypes: a populationbased study," Stroke, vol. 32, no. 12, pp. 2735-2740, 2001.

[2] R. C. S. Seet, P. A. Friedman, and A. A. Rabinstein, "Prolonged rhythm monitoring for the detection of occult paroxysmal atrial fibrillation in ischemic stroke of unknown cause," Circulation, vol. 124, no. 4, pp. 477-486, 2011.

[3] T. Rizos, A. Wagner, E. Jenetzky et al., "Paroxysmal atrial fibrillation is more prevalent than persistent atrial fibrillation in acute stroke and transient ischemic attack patients," Cerebrovascular Diseases, vol. 32, no. 3, pp. 276-282, 2011.

[4] R. G. Hart, L. A. Pearce, and M. I. Aguilar, "Meta-analysis: antithrombotic therapy to prevent stroke in patients who have nonvalvular atrial fibrillation," Annals of Internal Medicine, vol. 146, no. 12, pp. 857-867, 2007.

[5] H. P. Adams Jr., G. del Zoppo, M. J. Alberts et al., "Guidelines for the early management of adults with ischemic stroke: a guideline from the American Heart Association/American Stroke 
Association Stroke Council, Clinical Cardiology Council, Cardiovascular Radiology and Intervention Council, and the Atherosclerotic Peripheral Vascular Disease and Quality of Care Outcomes in Research Interdisciplinary Working Groups: the American Academy of Neurology affirms the value of this guideline as an educational tool for neurologists," Circulation, vol. 115, no. 20, pp. e478-e534, 2007.

[6] J. C. Barthélémy, S. Féasson-Gérard, P. Garnier et al., "Automatic cardiac event recorders reveal paroxysmal atrial fibrillation after unexplained strokes or transient ischemic attacks," Annals of Noninvasive Electrocardiology, vol. 8, no. 3, pp. 194-199, 2003.

[7] L. A. Sposato, F. R. Klein, A. Jáuregui et al., "Newly diagnosed atrial fibrillation after acute ischemic stroke and transient ischemic attack: importance of immediate and prolonged continuous cardiac monitoring," Journal of Stroke and Cerebrovascular Diseases, vol. 21, no. 3, pp. 210-216, 2012.

[8] R. Stahrenberg, M. Weber-Krüger, J. Seegers et al., "Enhanced detection of paroxysmal atrial fibrillation by early and prolonged continuous holter monitoring in patients with cerebral ischemia presenting in sinus rhythm," Stroke, vol. 41, no. 12, pp. 2884-2888, 2010.

[9] D. Jabaudon, J. Sztajzel, K. Sievert, T. Landis, and R. Sztajzel, "Usefulness of ambulatory 7-day ECG monitoring for the detection of atrial fibrillation and flutter after acute stroke and transient ischemic attack," Stroke, vol. 35, no. 7, pp. 1647-1651, 2004.

[10] A. H. Tayal, M. Tian, K. M. Kelly et al., "Atrial fibrillation detected by mobile cardiac outpatient telemetry in cryptogenic TIA or stroke," Neurology, vol. 71, no. 21, pp. 1696-1701, 2008.

[11] D. J. Miller, M. A. Khan, L. R. Schultz et al., "Outpatient cardiac telemetry detects a high rate of atrial fibrillation in cryptogenic stroke," Journal of the Neurological Sciences, vol. 324, no. 1-2, pp. 57-61, 2013.

[12] A. Bhatt, A. Majid, A. Razak, M. Kassab, S. Hussain, and A. Safdar, "Predictors of occult paroxysmal atrial fibrillation in cryptogenic strokes detected by long-term noninvasive cardiac monitoring," Stroke Research and Treatment, vol. 2011, Article ID 172074, 5 pages, 2011.

[13] L. Elijovich, S. A. Josephson, G. L. Fung, and W. S. Smith, "Intermittent atrial fibrillation may account for a large proportion of otherwise cryptogenic stroke: a study of 30-day cardiac event monitors," Journal of Stroke and Cerebrovascular Diseases, vol. 18, no. 3, pp. 185-189, 2009.

[14] N. Gaillard, S. Deltour, B. Vilotijevic et al., "Detection of paroxysmal atrial fibrillation with transtelephonic EKG in TIA or stroke patients," Neurology, vol. 74, no. 21, pp. 1666-1670, 2010.

[15] A. C. Flint, N. M. Banki, X. Ren, V. A. Rao, and A. S. Go, "Detection of paroxysmal atrial fibrillation by 30 -day event monitoring in cryptogenic ischemic stroke: the Stroke and Monitoring for PAF in Real Time (SMART) Registry," Stroke, vol. 43, no. 10, pp. 2788-2790, 2012.

[16] P. D. Ziegler, T. V. Glotzer, E. G. Daoud et al., "Incidence of newly detected atrial arrhythmias via implantable devices in patients with a history of thromboembolic events," Stroke, vol. 41, no. 2, pp. 256-260, 2010.

[17] V. Fuster, L. E. Rydén, D. S. Cannom et al., "ACC/AHA/ESC 2006 guidelines for the management of patients with Atrial Fibrillation: a report of the American College of Cardiology/American Heart Association Task Force on Practice Guidelines and the European Society of Cardiology Committee for Practice Guidelines (Writing Committee to Revise the 2001 Guidelines for the Management of Patients With Atrial
Fibrillation): developed in collaboration with the European Heart Rhythm Association and the Heart Rhythm Society," Circulation, vol. 114, no. 7, pp. e257-e354, 2006.

[18] W. M. Pollak, J. D. Simmons, A. Interian Jr. et al., "Clinical utility of intraatrial pacemaker stored electrograms to diagnose atrial fibrillation and flutter," Pacing and Clinical Electrophysiology, vol. 24, no. 4 I, pp. 424-429, 2001.

[19] C. Eitel, D. Husser, G. Hindricks et al., "Performance of an implantable automatic atrial fibrillation detection device: impact of software adjustments and relevance of manual episode analysis," Europace, vol. 13, no. 4, pp. 480-485, 2011.

[20] Z. Binici, T. Intzilakis, O. W. Nielsen, L. Køber, and A. Sajadieh, "Excessive supraventricular ectopic activity and increased risk of atrial fibrillation and stroke," Circulation, vol. 121, no. 17, pp. 1904-1911, 2010.

[21] T. V. Glotzer, E. G. Daoud, D. G. Wyse et al., “The relationship between daily atrial tachyarrhythmia burden from implantable device diagnostics and stroke risk: the TRENDS study," Circulation, vol. 2, no. 5, pp. 474-480, 2009.

[22] J. S. Healey, S. J. Connolly, M. R. Gold et al., "Subclinical atrial fibrillation and the risk of stroke," The New England Journal of Medicine, vol. 366, no. 2, pp. 120-129, 2012.

[23] A. M. Sinha, H. C. Diener, C. A. Morillo et al., "Cryptogenic Stroke and underlying Atrial Fibrillation (CRYSTAL AF): design and rationale," American Heart Journal, vol. 160, no. 1, pp. 36.el-41.el, 2010.

[24] J. Ip, A. L. Waldo, G. Y. H. Lip et al., "Multicenter randomized study of anticoagulation guided by remote rhythm monitoring in patients with implantable cardioverter-defibrillator and CRT$\mathrm{D}$ devices: rationale, design, and clinical characteristics of the initially enrolled cohort. The IMPACT study," American Heart Journal, vol. 158, no. 3, pp. 364.el-370.e1, 2009.

[25] D. Schwartzman, D. P. Blagev, M. L. Brown, and R. Mehra, "Electrocardiographic events preceding onset of atrial fibrillation: insights gained using an implantable loop recorder," Journal of Cardiovascular Electrophysiology, vol. 17, no. 3, pp. 243-246, 2006.

[26] R. Stahrenberg, F. Edelmann, B. Haase et al., "Transthoracic echocardiography to rule out paroxysmal atrial fibrillation as a cause of stroke or transient ischemic attack," Stroke, vol. 42, no. 12, pp. 3643-3645, 2011.

[27] D. Wallmann, D. Tüller, K. Wustmann et al., "Frequent atrial premature beats predict paroxysmal atrial fibrillation in stroke patients: an opportunity for a new diagnostic strategy," Stroke, vol. 38, no. 8, pp. 2292-2294, 2007.

[28] S. Malik, W. J. Hicks, L. Schultz et al., "Development of a scoring system for atrial fibrillation in acute stroke and transient ischemic attack patients: the LADS scoring system," Journal of the Neurological Sciences, vol. 301, no. 1-2, pp. 27-30, 2011.

[29] L. Suissa, D. Bertora, S. Lachaud, and M. H. Mahagne, "Score for the targeting of atrial fibrillation (STAF): a new approach to the detection of atrial fibrillation in the secondary prevention of ischemic stroke," Stroke, vol. 40, no. 8, pp. 2866-2868, 2009.

[30] E. I. Charitos, U. Stierle, P. D. Ziegler et al., "A comprehensive evaluation of rhythm monitoring strategies for the detection of atrial fibrillation recurrence: insights from 647 continuously monitored patients and implications for monitoring after therapeutic interventions," Circulation, vol. 126, no. 7, pp. 806-814, 2012.

[31] G. Hindricks, E. Pokushalov, L. Urban et al., "Performance of a new leadless implantable cardiac monitor in detecting 
and quantifying atrial fibrillation results of the XPECT trial," Circulation, vol. 3, no. 2, pp. 141-147, 2010.

[32] K. G. Haeusler, L. Koch, J. Ueberreiter et al., "Safety and reliability of the insertable Reveal XT recorder in patients undergoing 3 Tesla brain magnetic resonance imaging," Heart Rhythm, vol. 8, no. 3, pp. 373-376, 2011.

[33] H. Kamel, B. B. Navi, L. Elijovich et al., "Pilot randomized trial of outpatient cardiac monitoring after cryptogenic stroke," Stroke, vol. 44, no. 2, pp. 528-530, 2013.

[34] D. D. McManus, J. Lee, O. Maitas et al., "A novel application for the detection of an irregular pulse using an iPhone $4 \mathrm{~S}$ in patients with atrial fibrillation," Heart Rhythm, vol. 10, no. 3, pp. 315-319, 2013.

[35] P. T. Ellinor, K. L. Lunetta, C. M. Albert et al., "Meta-analysis identifies six new susceptibility loci for atrial fibrillation," $\mathrm{Na}$ ture Genetics, vol. 44, no. 6, pp. 670-675, 2012.

[36] J. G. Smith, C. Newton-Cheh, P. Almgren, O. Melander, and P. G. Platonov, "Genetic polymorphisms for estimating risk of atrial fibrillation in the general population: a prospective study," Archives of Internal Medicine, vol. 172, no. 9, pp. 742-744, 2012. 


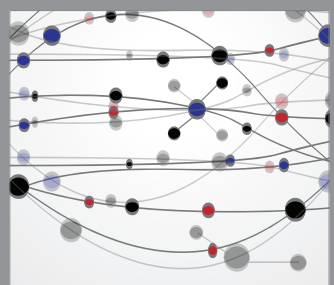

The Scientific World Journal
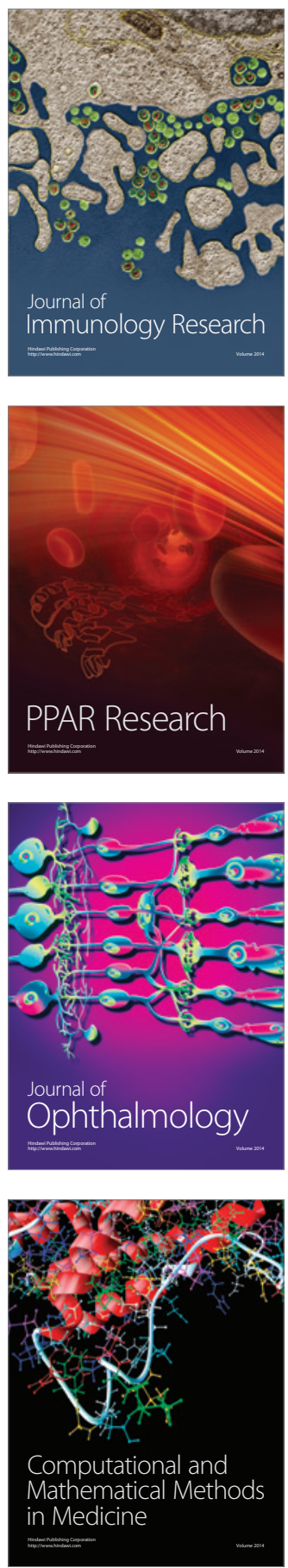

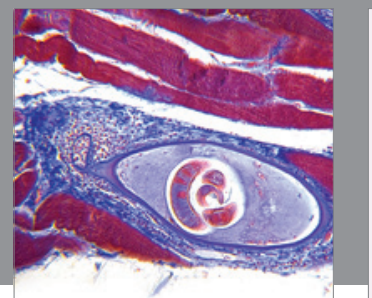

Gastroenterology

Research and Practice
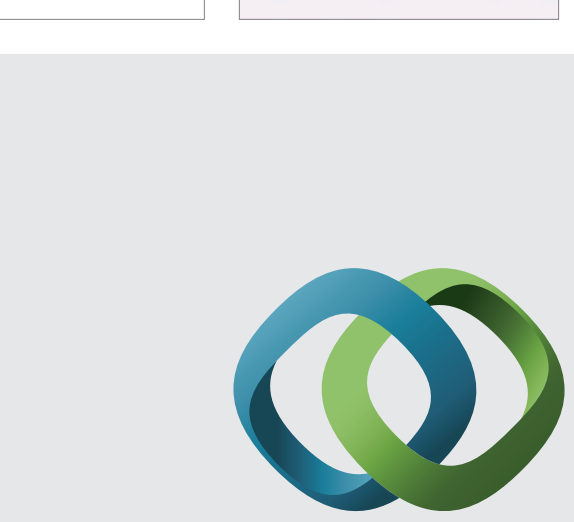

\section{Hindawi}

Submit your manuscripts at

http://www.hindawi.com
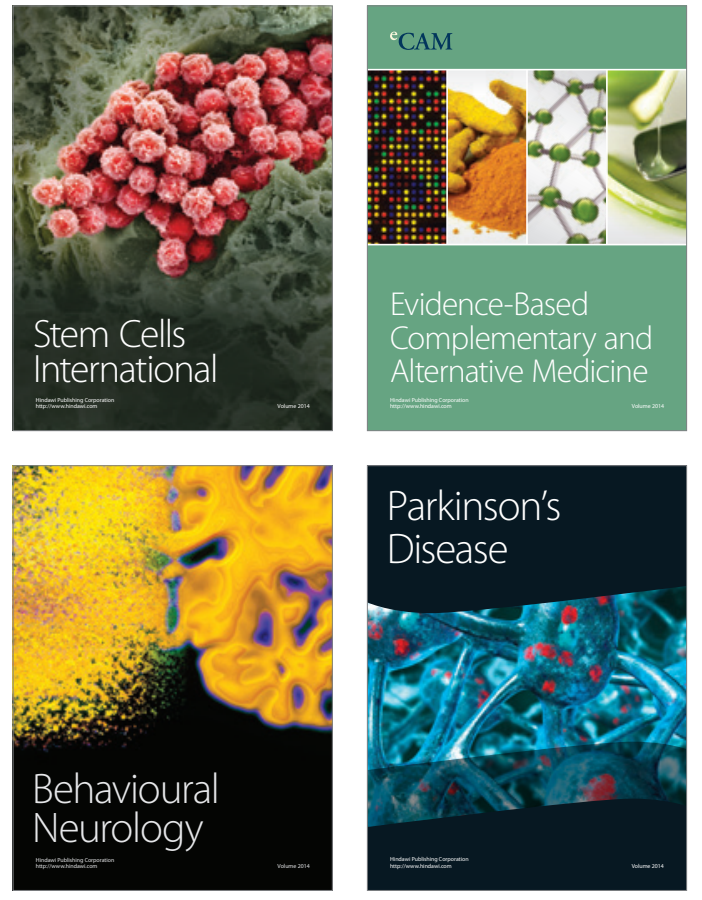
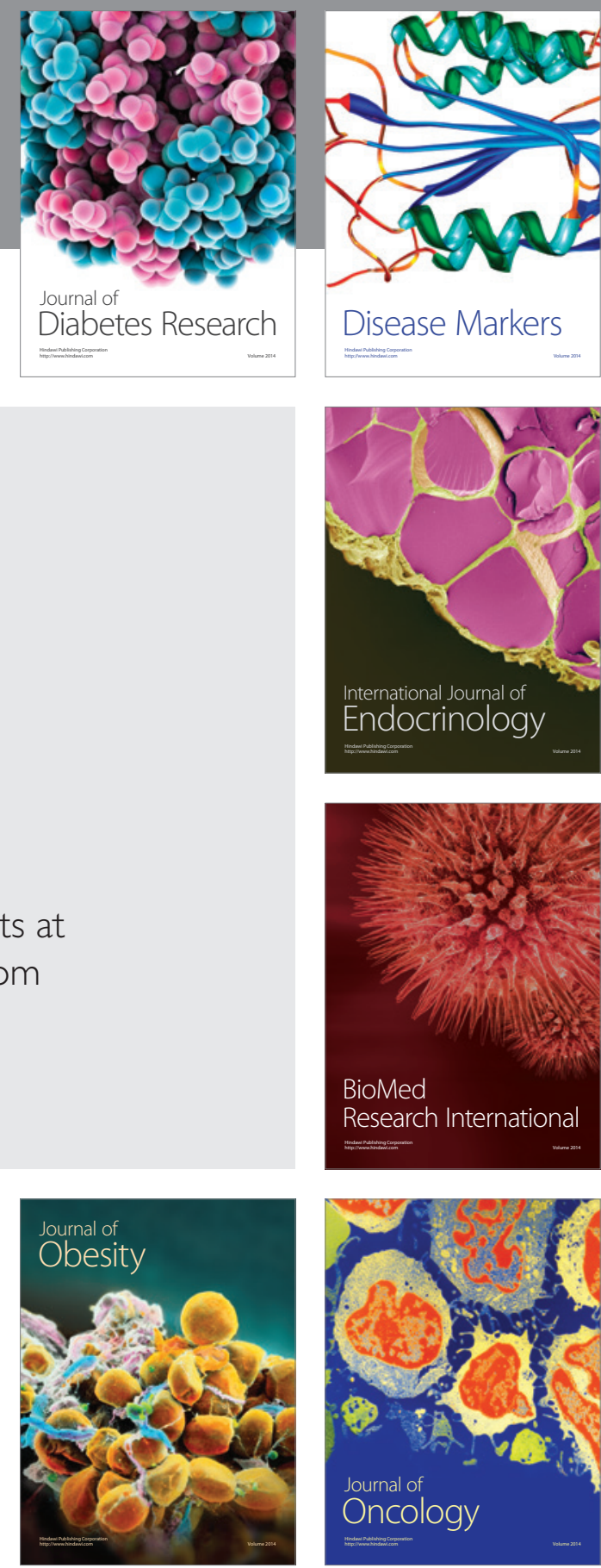

Disease Markers
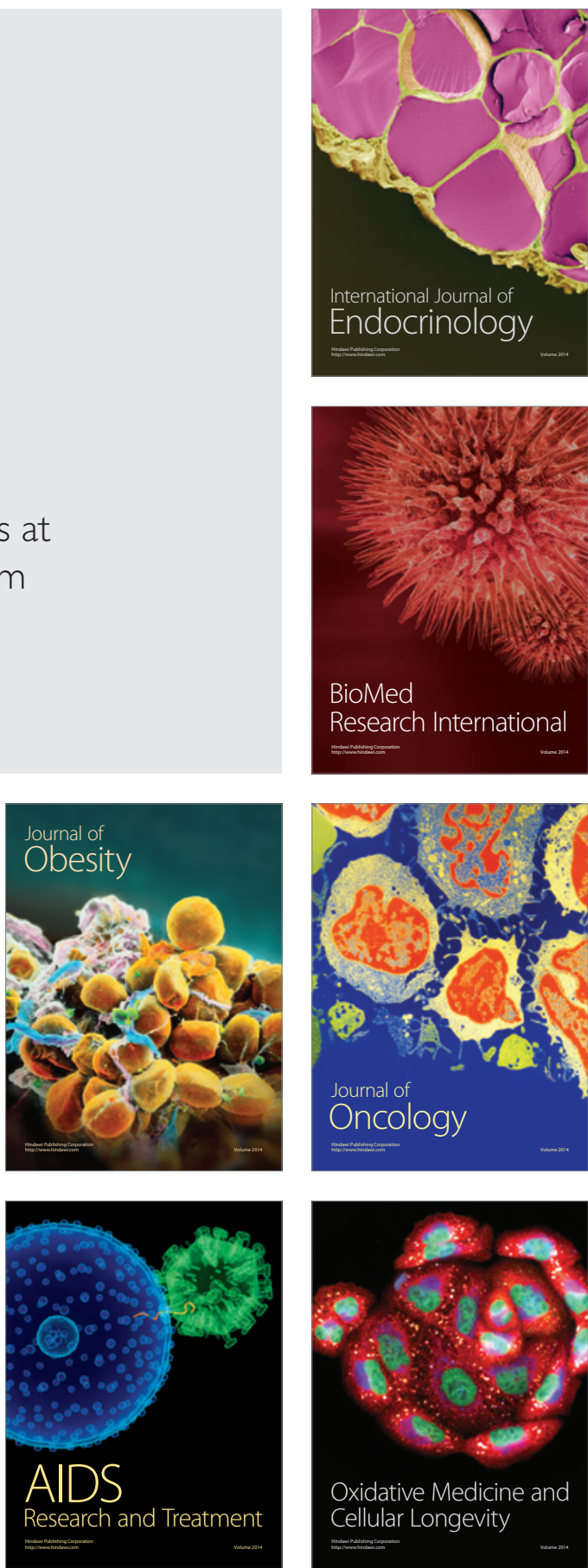